\title{
Numerical simulation of ballistic impact on composite laminates
}

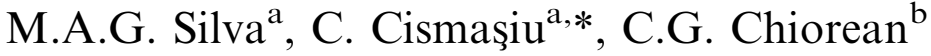 \\ ${ }^{a}$ Centro de Investigação em Estruturas e Construção - UNIC, Faculdade de Ciências e Tecnologia, Universidade Nova de \\ Lisboa, Quinta da Torre, Monte de Caparica 2829-516, Portugal \\ ${ }^{\mathrm{b}}$ Faculty of Civil Engineering, Technical University of Cluj-Napoca, 15 C. Daicoviciu Str., Cluj-Napoca 3400, Romania
}

Received 29 January 2003; received in revised form 29 November 2003; accepted 30 January 2004

\begin{abstract}
The paper reports experimental and numerical simulation of ballistic impact problems on thin composite laminated plates reinforced with Kevlar 29. Ballistic impact was imparted with simulated fragments designed in accordance with STANAG-2920 on plates of different thickness. Numerical modelling was developed and used to obtain an estimate for the limit perforation velocity $\left(V_{50}\right)$ and simulate failure modes and damage. Computations were carried out using a commercial code based on finite differences and values obtained are compared with the experimental data to evaluate the performance of the simulation. Good correlation between computational simulation and experimental results was achieved, both in terms of deformation and damage of the laminates. Future work is advanced to include the interposition of an outer ceramic layer as well as examining the influence of dry-wet and temperature cycles on the mechanical strength of the plates and their temporal evolution under accelerated ageing.

(C) 2004 Elsevier Ltd. All rights reserved.
\end{abstract}

Keywords: Non-linear transient dynamics; Ballistic impact; Composite materials

\section{Introduction}

The relatively high complexity of impact problems $[1,2]$ is caused by the large number of intervening parameters like relative velocity of projectile and target, shape of colliding objects, relative stiffness and masses, time-dependent surface of contact, geometry and boundary conditions and material characteristics. Difficulties increase when composite materials are involved, namely plates of FRP, due to orthotropy, larger variety of failure modes and

\footnotetext{
*Corresponding author.

E-mail address: cornel@fct.unl.pt (C. Cismaşiu).
} 
uncertainties on constitutive laws. Designing ballistic barriers of Kevlar 29 plates, based solely on physical models, would require a large number of experimental data, which are time consuming and costly to generate. Recent advances towards understanding damage mechanisms of laminated composites, (e.g. [3-5]) offer the possibility of avoiding many of the experimental tests by using computational simulation, bearing in mind that the numerical results should be used with precaution and be always certified by experimental tests.

One of the major problems posed to model adequately the phenomena lies on the constitutive equations, especially on strain rate effects. The tensile characteristics of composite laminates are difficult to determine [6] due to several factors that add to the usual difficulties to ensure adequate clamping that avoids stress concentrations and/or slipping of coupons at the jaws. Results for aramidic FRP are especially scarce, but the strain rate imposed by striking at a velocity around and above $400 \mathrm{~m} / \mathrm{s}$ leads to higher tensile strength and lower failure strain affecting the stressstrain law.

Important characteristics governing the behaviour of composite materials under ballistic impact are material anisotropy, coupling of volumetric and deviatoric behaviour, material compaction and phase changes, aspects addressed in the technical literature of recent years, (e.g. [7-9]). In the case of anisotropic materials, there is a strong coupling between the equation of state and the constitutive relations, as volumetric strain leads to deviatoric stress and similarly, deviatoric strain leads to spherical stress.

Failure occurs in a brittle manner, and is instantaneous in the specified failure directions. Postfailure material stiffness coefficients are assumed equal to those for the intact material in directions orthogonal to the failed direction.

There is a minimum threshold for the velocity that causes damage to start in form of delamination or matrix crazing. At higher velocities, composite cracking takes place and, for still higher impact velocity, partial or complete material break-out appears.

The objective of this paper is to report on experimental work and corresponding numerical simulation of impact problems on composite laminate plates reinforced with Kevlar 29 and to illustrate the performance of the simulation, laying ground for predictions solely based on numerical models.

All the simulations presented in the paper have been carried out using AUTODYN [10], a specially designed hydrocode for non-linear transient dynamic events such as ballistic impact, penetration and blast problems. The software is based on explicit finite difference, finite volume and finite element techniques, which use both grid based and gridless numerical methods. A new material model, specially designed for the shock response of anisotropic material is available. The model couples non-linear anisotropic constitutive relations with an equation of state. A set of partial differential equations for conservation of mass, momentum and energy is solved together with the constitutive equations using an explicit time integration scheme. An additional relationship between pressure, volume and energy completes a solvable set describing the purely hydrodynamic portions of stresses and strains. These, together with a material model and a set of initial and boundary conditions, define the complete solution of the problem. Impact is controlled by a so-called impact/slidelines procedure, allowing impacted material to erode, so that deep penetrations can be accounted for. The erosion is a numerical procedure which allows automatic removal of elements when they become heavily distorted. 


\section{Experimental programme}

In order to obtain the ballistic performance and $V_{50}$ value, a series of ballistic tests were performed on Kevlar 29/Vyniliester laminates plates at the Navy School in Lisbon. The plates, supplied by INEGI Porto, were made from prepreg fabric impregnated with vynilester resin and cured using the manufacturer's curing conditions.

The target in the ballistic experimental tests was a $400 \times 400 \mathrm{~mm}^{2}$ Kevlar 29/Vynilester plate, firmly clamped on the edges. Its $2.4 \mathrm{~mm}$ thickness correspond to seven layers. Each plate was impacted with four projectiles as shown in Fig. 1. The experimental locations chosen for projectile impact were selected so as to minimise the effects of previous impact damage and sufficiently far from the boundaries.

The target was impacted by a fragment simulating projectile (FSP). The FSPs are defined by STANAG-2920 and US MIL-P-46593 and manufactured according to MIL-P-46593A. They are cylindrical with a blunt chisel shaped nose and a raised flange at the base, as shown in Fig. 2.

The ballistic limit velocity, $V_{50}$, is defined as the velocity at which the projectile, striking a specified target with zero yaw and obliquity, has a $50 \%$ chance of perforating the target. Its value is estimated experimentally by shooting the targets across a broad range of velocities associated in our case with different amounts of loaded explosives. It is determined by taking the average of an equal number of highest partial penetration velocities and lowest complete penetration velocities which occur within a specific, relatively narrow, velocity range.

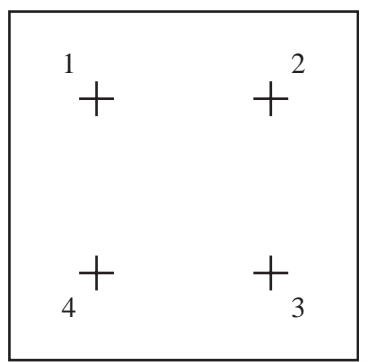

Fig. 1. Sketch of impact locations and firing order for specimen testing.

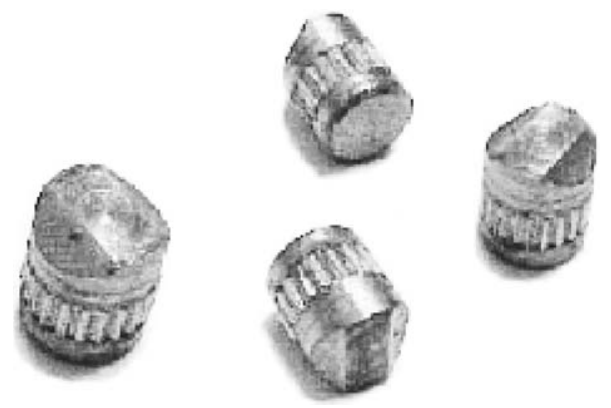

Fig. 2. Fragment-simulated projectiles. 
This method was followed to determine the $V_{50}$, varying the velocity of the fragment to achieve three perforations and three partial penetrations within a small velocity dispersion range of, typically, $38 \mathrm{~m} / \mathrm{s}$. The six velocities were then averaged to derive $V_{50}$ as illustrated in Fig. 3 .

Experimental tests reveal a number of damage mechanisms for composite laminated plates: there is a minimum velocity when damage occurs in the form of delamination or matrix crazing, followed by composite cracking at higher velocity, generally by fibre-matrix separation, moving to delamination associated with matrix cracking and/or matrix-fibre debonding, fibre failure and combined delamination and fibre failure. At velocities around $400 \mathrm{~m} / \mathrm{s}$, depending slightly on number of plies, shear-out of material takes place. Fig. 4 corresponds to impact at $320 \mathrm{~m} / \mathrm{s}$ and reveals a cruciform type of damage that evolves with velocity according to recognisable patterns.

In order to obtain the values of the mechanical material characteristics for numerical simulation, Ernst-Mach-Institut in Germany was engaged to perform quasi-static tensile tests on Kevlar 29/Vynilester laminates, see Fig. 5.

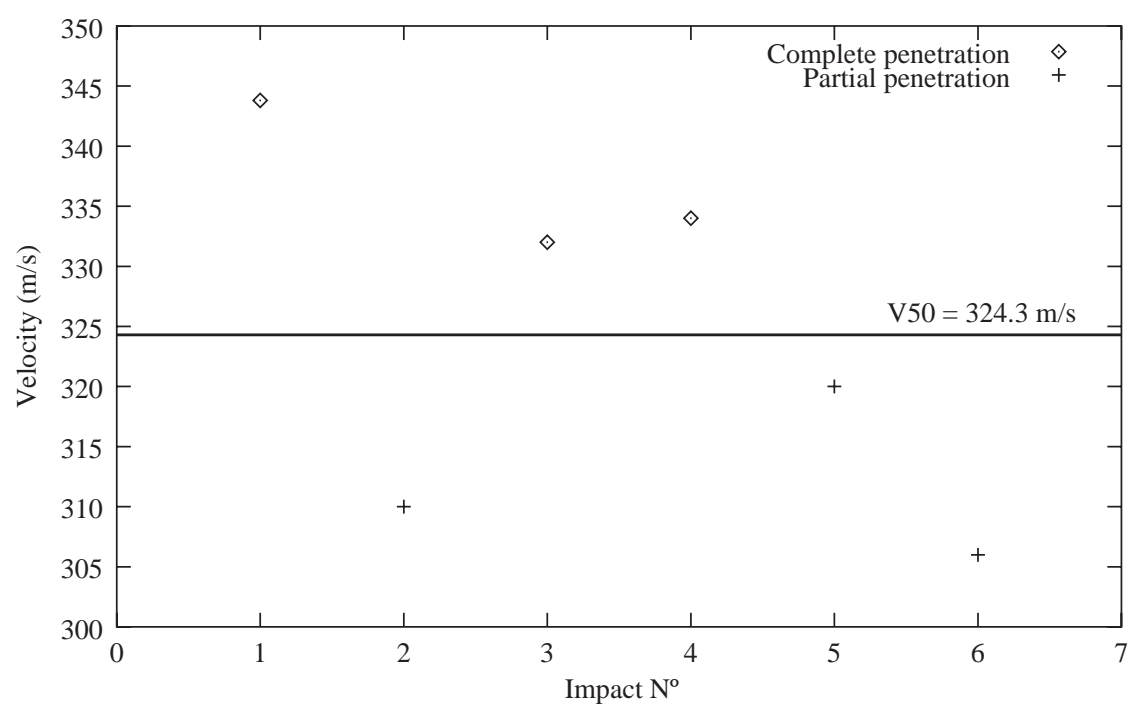

Fig. 3. Data points used to determine the military $V_{50}$ ballistic limit.

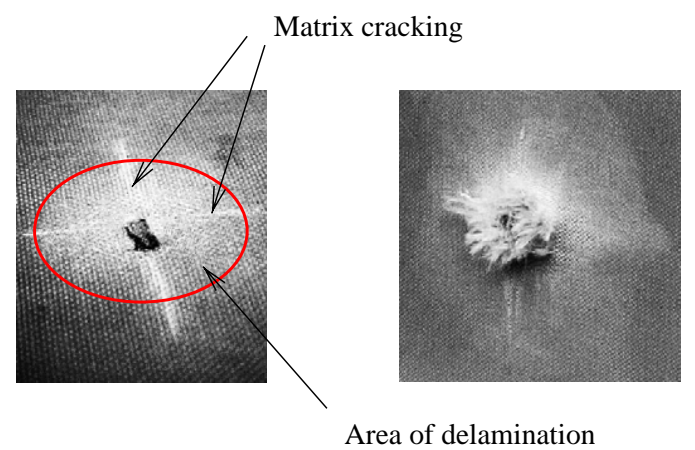

Fig. 4. Damage in laminated plates: front view and back view. 


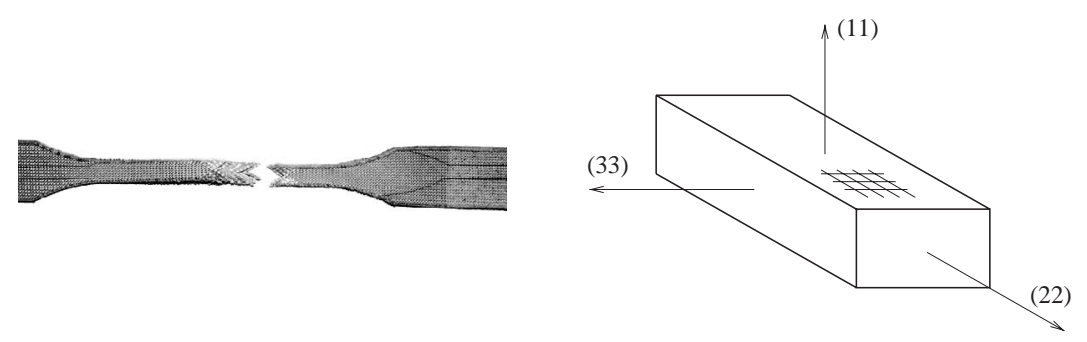

Fig. 5. Uniaxial static tension tests.

Table 1

EMI's orthotropic elastic properties

\begin{tabular}{|c|c|c|c|}
\hline No. of tests & Loading direction & Strain gauge & Results \\
\hline 3 & $\begin{array}{l}\text { Tensile } 0-22^{\circ} \\
\text { (in-plane) }\end{array}$ & $\begin{array}{l}22 \text { and } 23 \\
\text { on surface } \\
\text { (two channels) }\end{array}$ & $\begin{array}{l}E_{22 \text { machine }}=8075.95 \mathrm{MPa} \\
E_{22 \text { gauge }}=6311.69 \mathrm{MPa} \\
v_{23}=0.216\end{array}$ \\
\hline 3 & $\begin{array}{l}\text { Tensile } 45^{\circ}-22^{\circ} \\
\text { (in-plane) }\end{array}$ & $\begin{array}{l}\text { one channel } \\
\text { in } \\
\text { loading direction }\end{array}$ & $\begin{array}{l}E_{45^{\circ}}=3074.9 \mathrm{MPa} \\
v_{45^{\circ}}=0.77 \\
G_{23}=1540.54 \mathrm{MPa}\end{array}$ \\
\hline 3 & $\begin{array}{l}\text { Tensile } 0-22^{\circ} \\
\text { sample too thin }\end{array}$ & $\begin{array}{l}22 \text { and } 13 \text { through } \\
\text { thickness (two channels) }\end{array}$ & $\begin{array}{l}v_{13} \text { not possible } \\
\text { to measure }\end{array}$ \\
\hline 4 & Compression 11 & $\begin{array}{l}\text { one channel } \\
\text { circumferential }\end{array}$ & $\begin{array}{l}E_{11}=239.2 \mathrm{MPa} v_{13} \\
\text { not possible to measure }\end{array}$ \\
\hline
\end{tabular}

Quasi-static tensile tests were used to provide data on in-plane stiffness and failure strains. The through thickness stiffness $E_{11}$ was obtained in quasi-static compression tests. However, the fact that the sample thickness was around $2 \mathrm{~mm}$ and because of instantaneous through thickness delamination, EMI could not find Poisson's ratio $v_{13}$. The elastic constants obtained at EMI are presented in Table 1.

\section{Material model}

The most important characteristics and phenomena governing the behaviour of composite materials under ballistic impact are: material anisotropy, shock response (coupling of volumetric and deviatoric behaviour), anisotropic strength degradation, material compaction, phase changes. In the case of anisotropic materials, there is a strong coupling between the equation of state and the constitutive relations, as volumetric strain leads to deviatoric stress and similarly, deviatoric strain leads to spherical stress.

An advanced material model [8,9], specially designed to simulate the shock response of anisotropic materials has recently been implemented in AUTODYN [10]. As it is macro-mechanically 
based, the detailed micro-mechanics of the materials are neither represented in the numerical mesh nor in the material model. The model assumes that the composite material can be idealised as an orthotropic system. It is assumed that non-linear shock effects and associated energy dependency result from volumetric material strain. Deviatoric strain contributions to the final material pressure are based on a linear material response. The model includes also orthotropic brittle failure criteria to detect directional failure such as delamination.

Failure occurs in a brittle manner, and is instantaneous in the specified failure directions. Postfailure material stiffness coefficients are assumed equal to those for the intact material in directions orthogonal to the failed direction. The strain rate behaviour of Kevlar/Vinylester was considered more significant under compressive shock loading, which is well characterised in inverse flyer plate tests. It is expressed in the non-linear equation of state.

Therefore, static and dynamic tests have to be performed in order to obtain the mechanical characteristics of Kevlar/Vinylester materials. The confined static compression tests and the uniaxial static tension tests are used to derive the material stiffness, strength and compaction behaviour. Dynamic inverse flyer plate tests (IFPT) are required to obtain data on the behaviour of the material at high-strain rates.

Although the material model has been described previously [9], some of the key features will be mentioned briefly here because of their relevance to the analysis of laminated composite materials to high-velocity impact.

\subsection{Model features}

In an anisotropic material model, the traditional independent approach for the solution of the equation of state and constitutive relations is complicated because these two sub-models are strongly coupled: as stated before, volumetric strain leads to deviatoric stress, and similarly deviatoric strain leads to spherical stress. This interaction coupling is considered in the methodology proposed by Anderson et al. [11] and forms the basis of the model developed in [9]. Since for Kevlar/Vinylester one may not be able to consider the response as linear elastic, Anderson and his co-workers [11] suggest that the elastic constants in the stiffness matrix should be replaced by non-linear functions $C_{i j}$ which might depend on, for instance, strain. Further, so that the deviatoric and volumetric components can be coupled it is necessary to decompose the total strain tensor into volumetric $\left(\varepsilon_{\mathrm{V}}\right)$ and deviatoric $\left(\varepsilon_{i j}^{\mathrm{d}}\right)$ components.

$$
\left\{\begin{array}{c}
\Delta \sigma_{11} \\
\Delta \sigma_{22} \\
\Delta \sigma_{33} \\
\Delta \sigma_{23} \\
\Delta \sigma_{31} \\
\Delta \sigma_{12}
\end{array}\right\}=\left[\begin{array}{cccccc}
C_{11} & C_{12} & C_{13} & 0 & 0 & 0 \\
C_{21} & C_{22} & C_{23} & 0 & 0 & 0 \\
C_{31} & C_{32} & C_{33} & 0 & 0 & 0 \\
0 & 0 & 0 & C_{44} & 0 & 0 \\
0 & 0 & 0 & 0 & C_{55} & 0 \\
0 & 0 & 0 & 0 & 0 & C_{66}
\end{array}\right]\left\{\begin{array}{c}
\Delta \varepsilon_{11}^{\mathrm{d}}+\Delta \varepsilon_{\mathrm{V}} / 3 \\
\Delta \varepsilon_{22}^{\mathrm{d}}+\Delta \varepsilon_{\mathrm{V}} / 3 \\
\Delta \varepsilon_{33}^{\mathrm{d}}+\Delta \varepsilon_{\mathrm{V}} / 3 \\
\Delta \varepsilon_{23}^{\mathrm{d}} \\
\Delta \varepsilon_{31}^{\mathrm{d}} \\
\Delta \varepsilon_{12}^{\mathrm{d}}
\end{array}\right\} .
$$

Since the pressure is the average of the three direct stresses

$$
\Delta P=-\frac{1}{3}\left(\Delta \sigma_{11}+\Delta \sigma_{22}+\Delta \sigma_{33}\right)
$$


from the stress-strain relations (1) one obtains

$$
\begin{aligned}
\Delta P= & -\frac{1}{9}\left(C_{11}+C_{22}+C_{33}+2 C_{12}+2 C_{23}+2 C_{31}\right) \Delta \varepsilon_{\mathrm{V}} \\
& -\frac{1}{3}\left(C_{11}+C_{21}+C_{31}\right) \Delta \varepsilon_{11}^{\mathrm{d}}-\frac{1}{3}\left(C_{12}+C_{22}+C_{32}\right) \Delta \varepsilon_{22}^{\mathrm{d}} \\
& -\frac{1}{3}\left(C_{13}+C_{23}+C_{33}\right) \Delta \varepsilon_{33}^{\mathrm{d}} .
\end{aligned}
$$

The contribution to the pressure of volumetric and deviatoric components of strain is now clearly identified in Eq. (3).

For an isotropic Hookean material, the first term on the right-hand side is equivalent to a linear equation of state, whilst the remaining deviatoric strain terms would be zero.

The first term of Eq. (3) can therefore be used to define the volumetric (thermodynamic) response of an orthotropic material in which the effective bulk modulus of the material $K$, is defined by

$$
K=\frac{1}{9}\left(C_{11}+C_{22}+C_{33}+2 C_{12}+2 C_{23}+2 C_{31}\right) .
$$

For an orthotropic material, one can replace the first term in Eq. (3) by a Mie-Gruneisen equation of state. The remaining terms act as a correction due to deviatoric strains.

Three Mie-Gruneisen forms of equation of state are available for coupling with an orthotropic response in the model: shock equation of state, linear equation of state and polynomial equation of state. The volumetric response of the material is defined through the solid equation of state. The polynomial sub-equation of state (5) allows non-linear shock effects to be coupled with the orthotropic material stiffness.

$$
P=A_{1} \varepsilon_{\mathrm{V}}+A_{2} \varepsilon_{\mathrm{V}}^{2}+A_{3} \varepsilon_{\mathrm{V}}^{3} .
$$

The first term in Eq. (5) is equivalent to a linear equation of state with the bulk modulus $K$ derived from orthotropic material stiffness coefficients.

\subsection{Damage of plates}

Composite materials of polymeric matrix subjected to impact exhibit complex behaviours. Experimentally, the dominant tensile material failure modes were identified as extensive delamination, due to matrix cracking and/or matrix-fibre debonding, in-plane fibre failure and punching shear failure caused by a combination of delamination and fibre failure leading to bulk failure. In the numerical model the composite material is considered to be homogeneous, i.e. Kevlar fibres and epoxy matrix are not separately modelled and the main phenomena of relevance are accounted for in a macro-mechanical model.

The incremental constitutive relations are assumed to be of the following form:

$$
\left\{\begin{array}{c}
\Delta \sigma_{11} \\
\Delta \sigma_{22} \\
\Delta \sigma_{33} \\
\Delta \sigma_{23} \\
\Delta \sigma_{31} \\
\Delta \sigma_{12}
\end{array}\right\}=\left[\begin{array}{cccccc}
C_{11} & C_{12} & C_{13} & 0 & 0 & 0 \\
C_{21} & C_{22} & C_{23} & 0 & 0 & 0 \\
C_{31} & C_{32} & C_{33} & 0 & 0 & 0 \\
0 & 0 & 0 & \alpha C_{44} & 0 & 0 \\
0 & 0 & 0 & 0 & \alpha C_{55} & 0 \\
0 & 0 & 0 & 0 & 0 & \alpha C_{66}
\end{array}\right]\left\{\begin{array}{c}
\Delta \varepsilon_{11} \\
\Delta \varepsilon_{22} \\
\Delta \varepsilon_{33} \\
\Delta \varepsilon_{23} \\
\Delta \varepsilon_{31} \\
\Delta \varepsilon_{12}
\end{array}\right\}
$$


assuming that the effect of the additional off diagonal terms for the monoclinic relations are of second-order importance for ballistic impact response.

In the incremental constitutive relations (6), delamination is assumed to result from excessive through-thickness tensile stresses or strains and/or from excessive shear stresses or strains in the matrix material.

The stress normal to the laminate and the corresponding orthotropic stiffness coefficients $C_{i j}$ are instantaneously set to zero, whenever the failure is initiated in either of those two modes, $j=1$ in

$$
\Delta \sigma_{i i}=0 \quad \text { and } \quad C_{i j}=C_{j i}=0 \quad \text { for } j=1,3 .
$$

Delamination may also result from reduction in shear stiffness of the composite, via parameter $\alpha$ in Eq. (6).

In-plane fibre failure is assumed to result from excessive stresses and/or strains in the 22 or 33 directions, $j=2$ or 3 in Eq. (7).

The combined effect of failure in all three material directions is represented by changing the material stiffness and strength to an isotropic characterisation, with no stress deviators or material tensile stresses. A fractional residual shear stiffness is maintained through the parameter $\alpha$, whose value must be obtained by experimental tests.

Composite material cell failure initiation criteria is based on a combination of material stress and strain failure. Subsequent to failure initiation, the cell stiffness and strength properties are modified in agreement with the failure initiation modes.

\subsection{Material data}

The input data for the numerical model of Kevlar 29/Vinylester material has been derived from experimental tests carried out at Ernst-Mach-Institut in Germany and through numerical calibration.

The non-linear equation of state parameter was derived iteratively through numerical calibration. Impact simulations were performed varying the value of the parameter until the results matched the experiments for all range of impact velocities.

Ernst-Mach-Institut achieved quasi-static tensile tests on Kevlar/Vinylester laminates in order to establish the material properties leading to the results shown in Table 1.

The positiveness of the stiffness $\boldsymbol{C}$ and the compliance $\boldsymbol{S}=\boldsymbol{C}^{-1}$ tensors in anisotropic materials is imposed by thermodynamic principles based on the fact that the elastic potential should remain always a positive quantity. The positive definiteness of these two tensors for the transversely isotropic materials implies that the following system of relations must hold [12]:

$$
\left|v_{12}\right|=\left|v_{13}\right|<\left(\frac{E_{11}}{E_{22}}\right)^{1 / 2}, \quad\left|v_{23}\right|<1
$$

and

$$
v_{12}^{2} v_{23}<\left(1-v_{23}^{2}\right) \frac{E_{11}}{2 E_{22}}-v_{12}^{2} .
$$

It can readily be derived from these relations in conjunction with the material data presented in Table 1, that the extreme limit of $v_{12}$ Poisson's ratio is 0.1219 . A high-velocity impact 
calibration was performed with $v_{12}$ Poisson's ratio ranging from zero to its maximum allowable value.

It is well-known that ballistic performance is dominated by the strength properties of the plate rather than its stiffness properties, fact confirmed by the ballistic response which proved to be less sensitive to this parameter. However, as results from the numerical simulations, the value of the Poisson ratio has a strong influence on failure initiation, propagation and pattern of damage. The value $v_{12}=0.08$ was found to be the one which produced a pattern of damage in the simulated tests which best matched the experimental tests for all range of impact velocities.

\subsubsection{Inverse flyer plate simulations}

In order to characterise the response of Kevlar/Vinylester at high strain rates and, in particular, to study the through thickness response, bi-dimensional IFPT were conducted numerically using AUTODYN 2D.

The flyer consists of a Kevlar element backed by a metallic plate (C45 steel) whilst the target witness plate is $\mathrm{C} 45$ steel. The numerical model used the Lagrange processor and planar symmetry was considerated. Uniaxial strain conditions were assumed and enforced through fixed velocity boundary conditions transverse to the impact direction. A single cell was used in the transverse direction with 20 cells through the thickness of material. Interaction between each component in the model was achieved using AUTODYN's Lag/Lag interaction logic [10].

The numerical simulations were conducted using the polynomial and linear sub-equation of state, respectively.

When the polynomial equation of state was used, four values for $A_{2}$ coefficient in the subequation of state (5) were considered: $0, K, 10 K$ and $100 K$, where $K$ is the orthotropic bulk modulus of material defined by Eq. (4), while keeping $A_{3}=0$.

The simulated back-surface velocities for $320 \mathrm{~m} / \mathrm{s}$ impact velocity are shown in Fig. 6. The IFPT simulation results obtained using a linear equation of state proved to be quite different

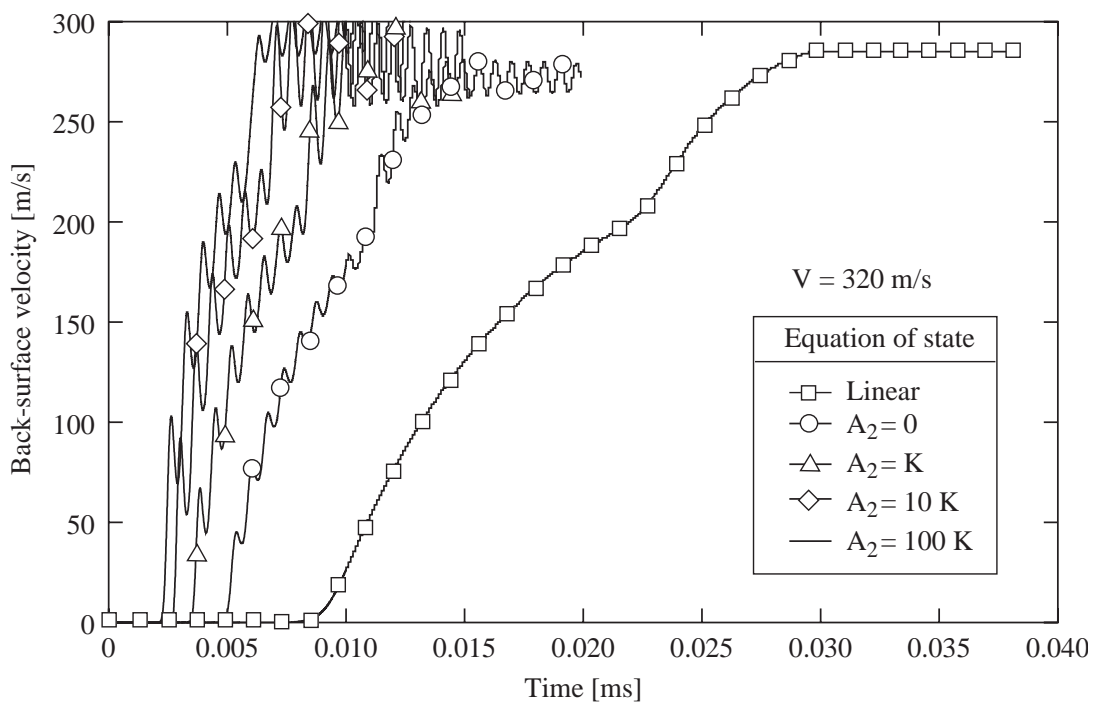

Fig. 6. IFPT simulation-impact velocity $320 \mathrm{~m} / \mathrm{s}$. 


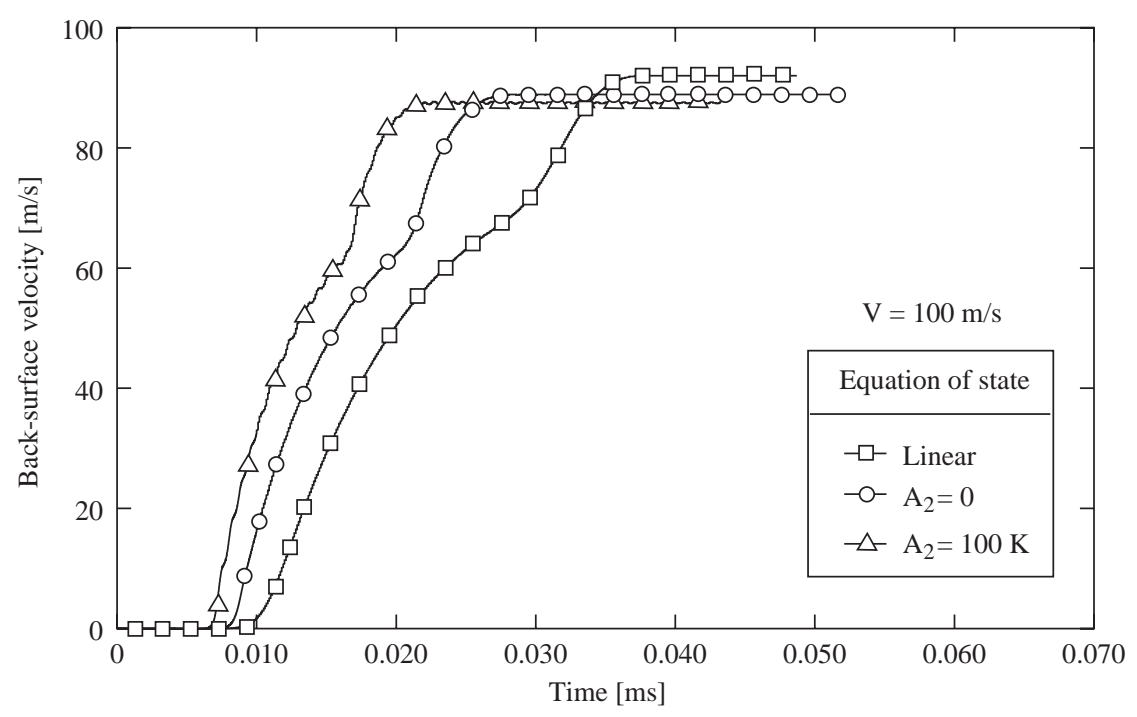

Fig. 7. IFPT simulation-impact velocity $100 \mathrm{~m} / \mathrm{s}$.

when compared with the ones obtained using a polynomial equation of state to define the volumetric response of material.

This difference decreases with velocity, as illustrated in Fig. 7. For an impact velocity of $100 \mathrm{~m} / \mathrm{s}$ the numerical simulations exhibit close agreement between linear and polynomial equation of state.

However, once the polynomial equation of state is used, the results proved to be less sensitive to $A_{2}$ and therefore a value of $10 \mathrm{~K}$ was adopted for future computations.

\section{Numerical simulations}

The projectile in all numerical simulations reported here is a 4340 steel STANAG-2920 fragment, modelled using a Johnson-Cook strength model, including strain and strain rate hardening and thermal softening effects.

The real target is a $400 \times 400 \mathrm{~mm}^{2}$ Kevlar 29/Vynilester plate, firmly clamped on the edges. As the high-velocity impact phenomenon is of localised nature, only a region of the real plate was modelled. Absorbing boundaries [13] were applied on lateral edges in order to avoid stress waves reflections. However, as the absorbing boundaries are not perfect transmitters of all waveforms, they should be placed at some reasonable distance from the region of main interest. A $100 \times$ $100 \mathrm{~mm}^{2}$ region proved to be sufficiently large to obtain the solution without significant perturbations due to reflected waves.

Taking into account the symmetry, the finite element mesh for both the target and the projectile was generated using TrueGrid [14]. The analysis starts with the impactor and plate in contact, as illustrated in Fig. 8 . 

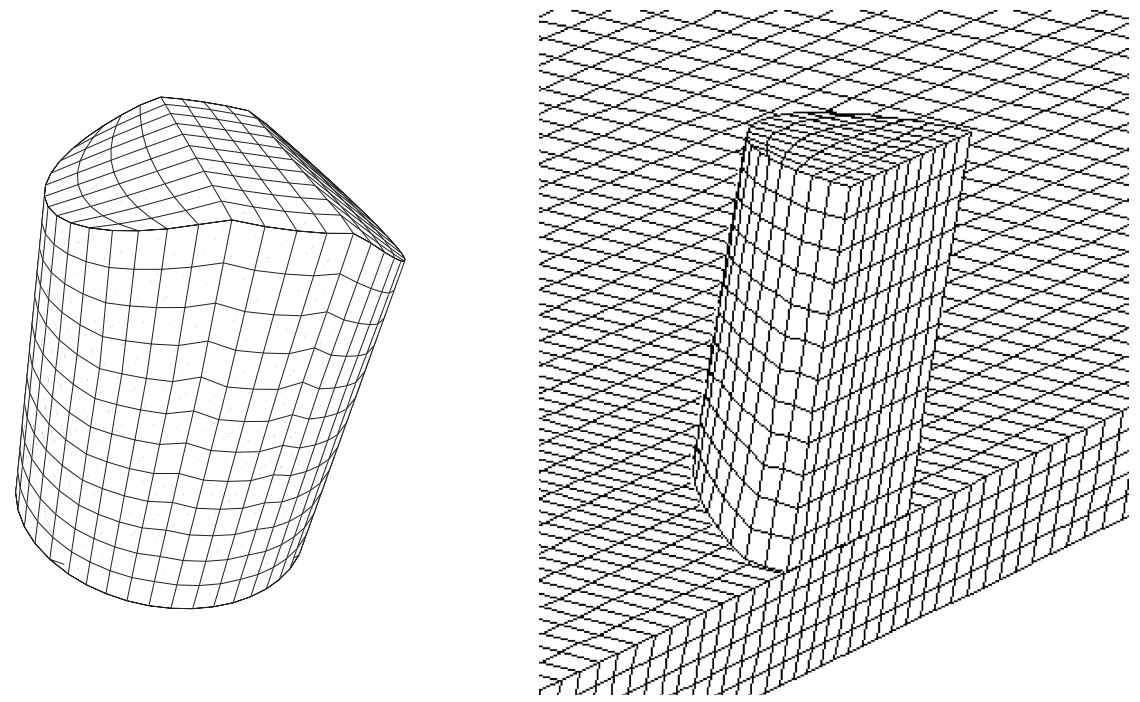

Fig. 8. Projectile view and contact at initial step.

Both the projectile and the composite target were modelled using a Lagrange processor, which means that the mesh is attached to the material and therefore they deform together. Cubic cells of $0.4 \mathrm{~mm}$ were used to discretise the target and projectile in the central impact area. Cells dimension gradually increased to the outer edges. During the analysis, degenerate cells were eroded at an instantaneous geometric strain equal to 1.5. Interaction between the projectile and the laminate target was handled by the impact logic, using a gap size $0.014 \mathrm{~mm}$. A broad range of impact velocities was tested in order to determine the ballistic limit and damage pattern.

\subsection{Comparison of results}

The ability of the numerical model to predict the response of laminated composite plates to ballistic impact depends largely on the selection of appropriate material models and associated input data.

The data referring the elastic moduli and the failure stresses/strains were obtained from quasistatic tensile tests conducted at Ernst-Mach-Institut. However, the response of Kevlar/Vinylester at high-strain rates and in particular the through thickness response, needs more data which can be obtained experimentally in IFPT. A set of numerical simulations should be compared with experimental results to tune up the equation of state. As such experimental data were not available, and taking into account the relatively small sensitivity of the results to $A_{2}$ parameter in the sub-equation of state (5), a value $A_{2}=10 \mathrm{~K}$ was adopted.

The influence of the type of the equation of state over the damage pattern can be see in Fig. 9. When compared with the experimental data, see Fig. 4, the results obtained using a linear equation of state are quite poor. When a polynomial sub-equation of state was selected, the results exhibit a significant improvement, and the matrix cracking effect, which has an important role in delamination propagation, can be captured. 
Another set of numerical simulations was performed in order to predict the ballistic limit and $V_{50}$. Experimental results reported in [15], indicate a ballistic limit of $324.3 \mathrm{~m} / \mathrm{s}$. Attempting to obtain this value, the target was subjected to an impact with increasing velocity. The history of the projectile velocity was recorded, as illustrated in Fig. 10 for an impact velocity of 320, 330, 340 and $360 \mathrm{~m} / \mathrm{s}$.

Analysing the plots from Fig. 10, one can conclude that the residual velocities are 170, 155 and $70 \mathrm{~m} / \mathrm{s}$ for impact velocities of 360,340 and $330 \mathrm{~m} / \mathrm{s}$, respectively. Since in the experimental tests these residual velocities were not measured, no comparison can be made. However, for an impact velocity of $320 \mathrm{~m} / \mathrm{s}$, the residual velocity is very low, but negative, which corresponds to the situation in which the bullet is rejected by the plate. Therefore, one can conclude that the ballistic limit, defined as the maximum velocity at which the projectile can be stopped [16] and which in the numerical simulations is equal to the limit with $V_{50}$, is $320 \mathrm{~m} / \mathrm{s}$. This result is in close agreement with the one obtained in experimental tests and reported in [15].

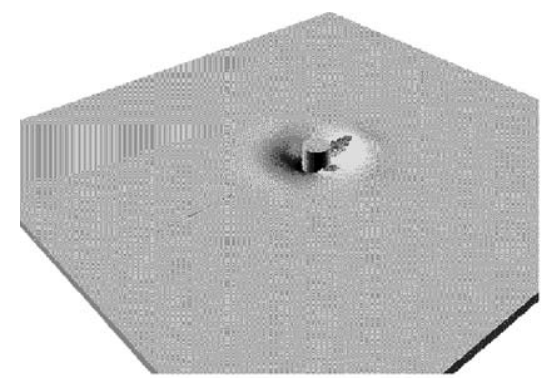

Linear equation of state

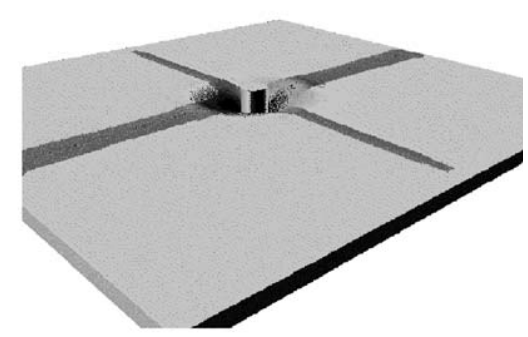

Polynomial sub-equation of state

Fig. 9. Simulated initial damage pattern.

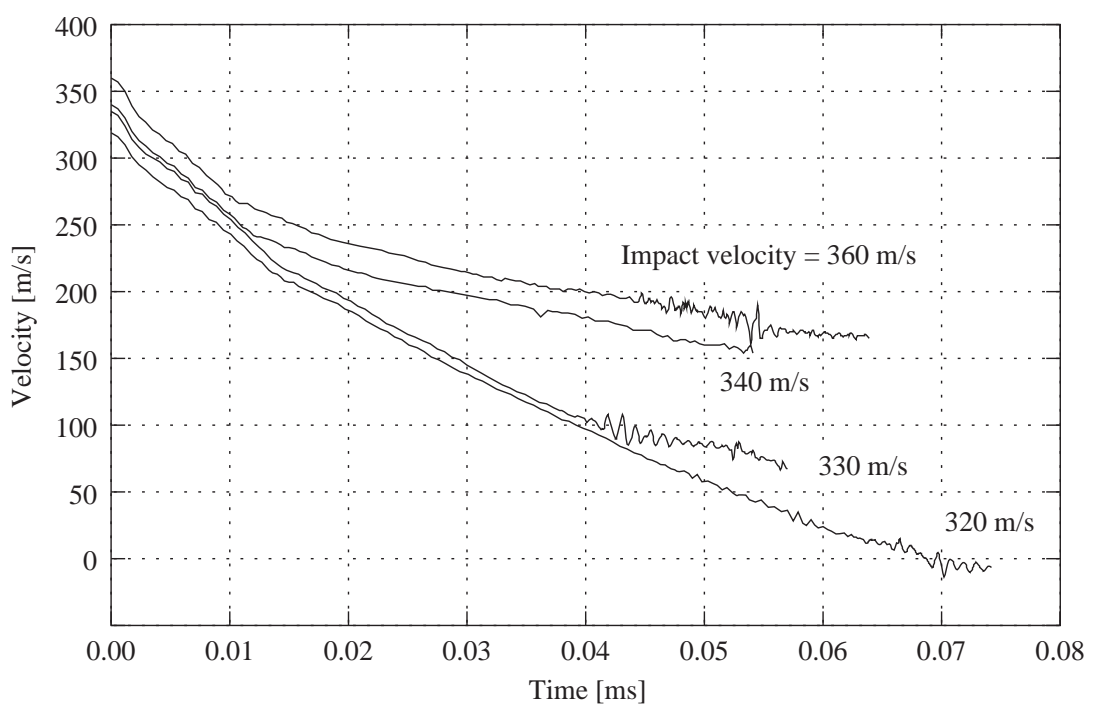

Fig. 10. Simulated projectile tail velocities. 
The damage propagation was also simulated. For an impact velocity of $320 \mathrm{~m} / \mathrm{s}$ the damage pattern was recorded during $63.51 \mu$ s, which corresponds to 13500 computational steps. These data are presented in Fig. 11.
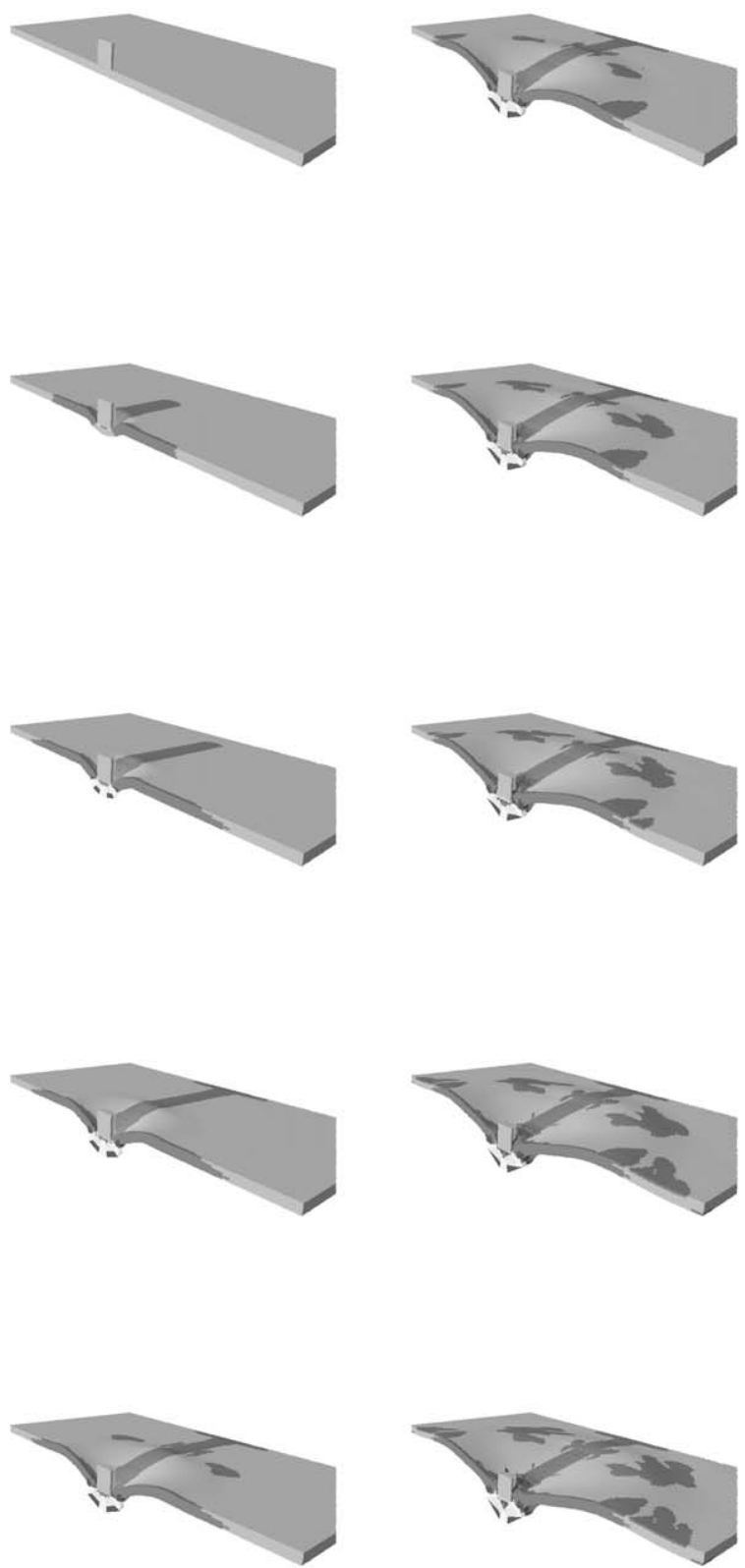

Elastic Plastic Bulk Fail Failed 11 Failed 22 Failed 33

Fig. 11. Simulated damage propagation. 
The initiation of the matrix cracking can be observed, followed by delamination and fibre failure in the last stages. The propagation of the stress waves can also be detected and the extension of the finite element mesh proves to be adequate as no reflected waves arrive to the impact zone during the penetration.
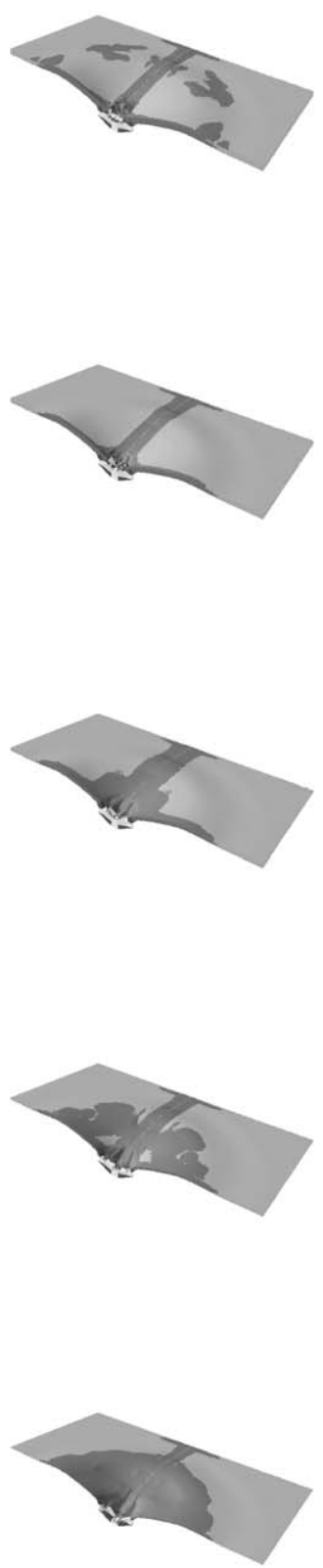

Elastic Plastic Bulk Fail Failed 11 Failed 22 Failed 33

Fig. 12. Damage through thickness. 
The evolution of the delamination through thickness, at instant $52.37 \mu$ s is presented in Fig. 12 for five layers. The first layer is the top surface where the impact occurs. The matrix cracking is evident as well as the stress wave which travels through the boundary together with a reflected wave which starts to appear at the boundary. In the next layer, situated at a $0.4 \mathrm{~mm}$ depth, the matrix cracking is still predominant. The third layer, at a $0.8 \mathrm{~mm}$ depth, still exhibits matrix cracking but the delamination becomes larger. At a $1.2 \mathrm{~mm}$ depth, the fourth layer presents larger delaminated area. The damage is practically due exclusively to delamination in the last layer, at $2 \mathrm{~mm}$ from the top surface and $0.4 \mathrm{~mm}$ from the back surface.

The area of the total damage obtained in the numerical simulations is compared with the experimental tests performed at Navy School and reported in [15]. As illustrated in Fig. 13, experimentally, delamination exhibits a circular shape of $95 \mathrm{~cm}^{2}$. In the numerical simulation the same shape was obtained with a delaminated area of $113 \mathrm{~cm}^{2}$.

The performance of the numerical simulation with the adopted material model was tested also when the impact velocity was grossly increased up to $1270 \mathrm{~m} / \mathrm{s}$. In this case, the target is a nine layer Kevlar 29/Vinylester plate of $3.0 \mathrm{~mm}$ thickness. The experimental residual velocity was now available and therefore could be compared with the one obtained in the simulation.

Front view: experiment vs. simulation
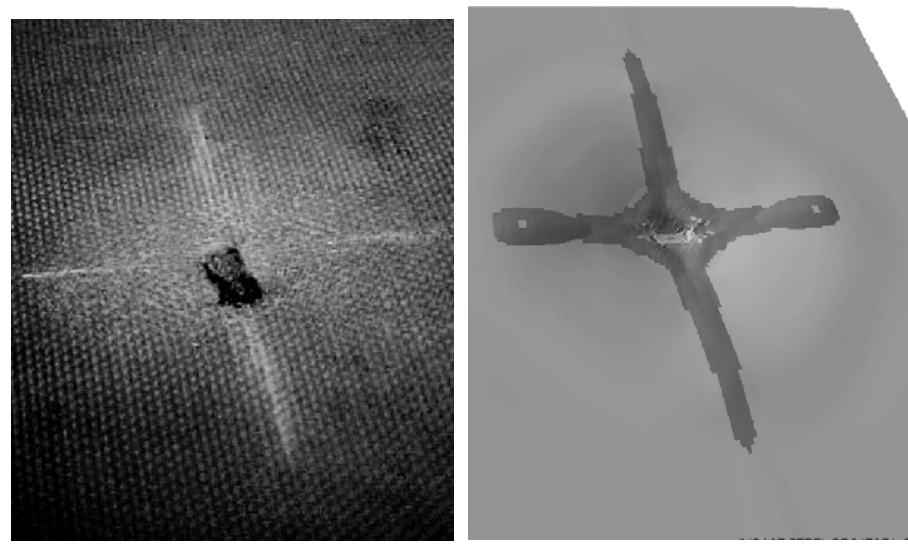

Back view: experiment vs. simulation
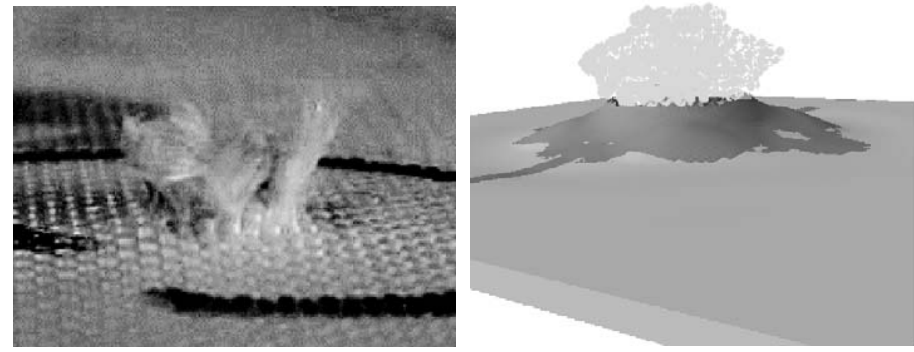

Fig. 13. Impact at $320 \mathrm{~m} / \mathrm{s}$ - total damage. 


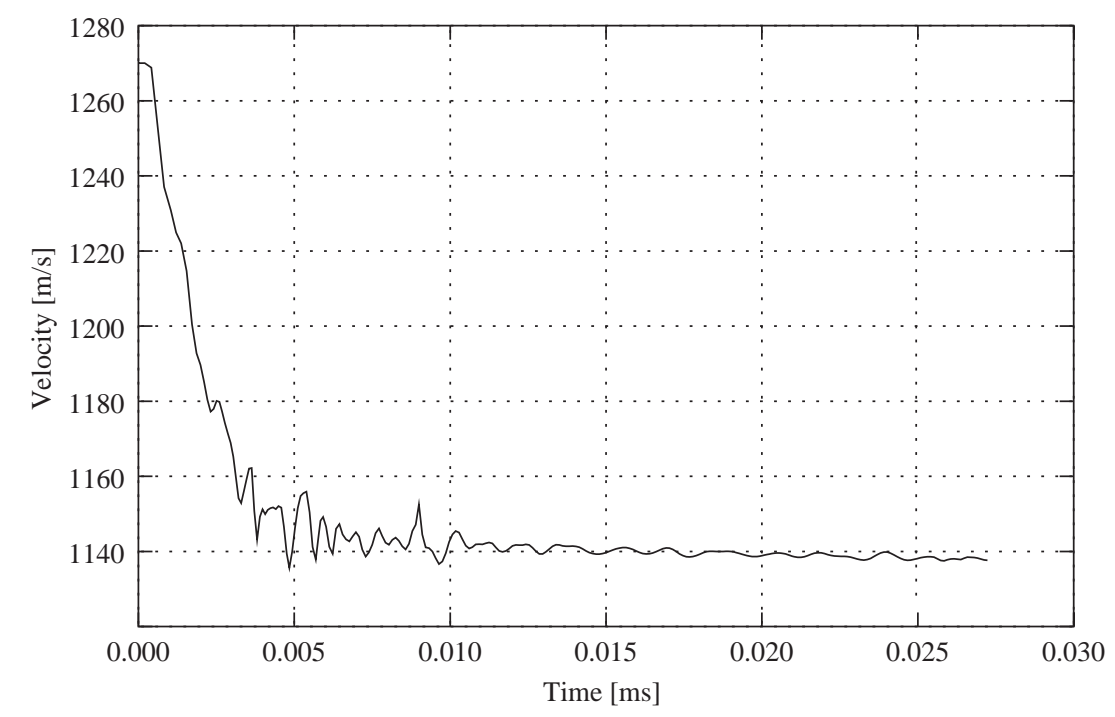

Fig. 14. Simulated projectile tail velocity-impact velocity $1270 \mathrm{~m} / \mathrm{s}$.

The projectile tail velocity during impact simulation was recorded and is presented in Fig. 14 . One can see that the residual velocity is about $1140 \mathrm{~m} / \mathrm{s}$, which is in close agreement with the experimental residual velocity, $1144 \mathrm{~m} / \mathrm{s}$.

As presented in Fig. 15, the total damage obtained in the simulated impact exhibits the same circular shape but with a much less extension when compared with lower impact velocities. The delaminated area - dark region - of $2.1 \mathrm{~cm}$ radius compares well with the global damage area obtained experimentally, of $2.25 \mathrm{~cm}$ radius.

\section{Final considerations}

An experimental study on ballistic impact on composite laminate plates reinforced with Kevlar 29/Vinylester was conducted at Navy School in Lisbon. The data resulting from this study was used to tune up an advanced model for orthotropic materials [8]. Its main advantage is the ability to use a non-linear equation of state in conjunction with an orthotropic stiffness matrix which allows an accurate modelling of the response of composite materials under impact conditions.

The ballistic impact of a simulated fragment on Kevlar 29/Vinylester fibre reinforced panel was simulated based on the calibrated material model, using the finite difference hydrocode AUTODYN [10].

The estimates for $V_{50}$, global damage and residual velocity were very accurate when compared with the experimental data.

These results encourage the use of computer simulation in the design of ballistic light armours in order to avoid expensive and time-consuming experiments.

Future work is envisaged considering the interposition of a ceramic type layer and oblique impact. 


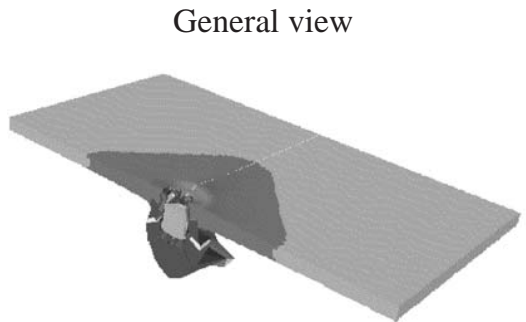

Frontview
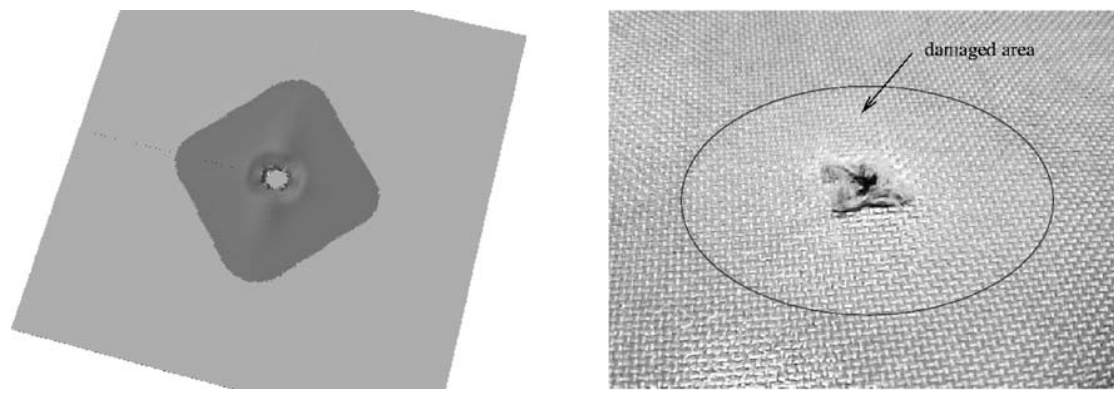

Backview
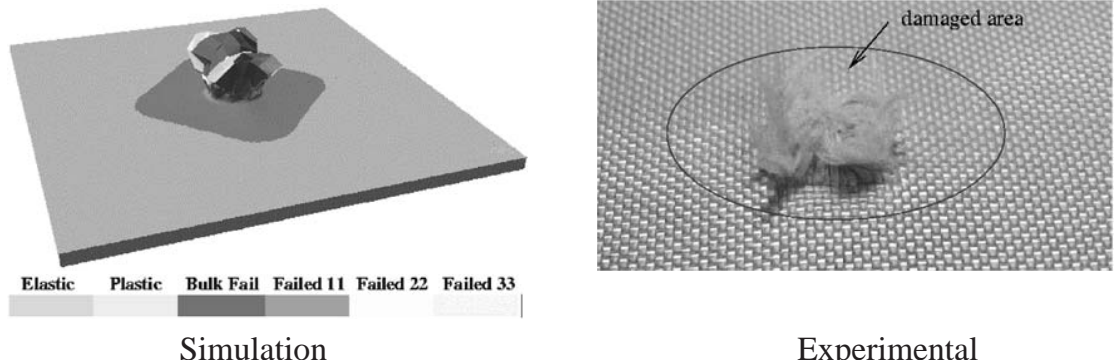

Simulation

Experimental

Fig. 15. Simulated vs. experimental damage for an impact velocity of $1270 \mathrm{~m} / \mathrm{s}$.

\section{Acknowledgements}

This work is part of the research developed at DEC, Faculdade de Ciências e Tecnologia, supported by contract 43228/EME/2001 with Fundação para a Ciência e Tecnologia. Cooperation with colleagues from INEGI, Porto and Comd. F. Neto from Navy School of Lisbon is gratefully acknowledged.

\section{References}

[1] Smith PD, Hetherington JG. Blast and ballistic loading of structures. London: Butterworth-Heinemann Ltd.; 1994.

[2] Abrate S. Impact on composite structures. Cambridge: Cambridge University Press; 1998. 
[3] Choi H, Downs R, Chang F. A new approach toward understanding damage mechanisms and mechanics of laminated composites due to low-velocity impact: Part I-experiments. J Comp Mater 1991;25:992-1011.

[4] Choi H, Downs R, Chang F. A new approach toward understanding damage mechanisms and mechanics of laminated composites due to low-velocity impact: Part II—analysis. J Comp Mater 1991;25:1012-38.

[5] Choi H, Chang F. A model for predicting damage in graphite/epoxy laminated composites resulting from lowvelocity point impact. J Comp Mater 1992;26(14):2134-69.

[6] Silva MAG. Low speed impact on polyethilene and aramidic FRP laminates. In: Proceedings of Conference on Integrity, Reliability and Failure, Porto, 1999.

[7] Clegg R, Hayhurst C, Leahy J, Deutekom M. Application of coupled anisotropic material model to high velocity impact response of composite textile armour. In: 18th International Symposium and Exhibition on Ballistics, San Antonio, TX, USA, 1999.

[8] Hayhurst C, Hiemaier S, Clegg R, Riedel W, Lambert M. Development of material models for nextel and kevlarepoxy for high pressures and strain rates. In: Hypervelocity Impact Symposium, Huntsville, AL, 1999.

[9] Hiermaier S, Riedel W, Clegg R, Hayhurst C. Advanced material models for hypervelocity impact simulations. Technical Report, ESA/ESTEC Contract No. 12400/97/NL/PA(SC), 1999.

[10] Century Dynamics, Inc., AUTODYN. Interactive non-linear dynamic analysis software, Horsam, UK: Century Dynamics, Ltd; 1997.

[11] Anderson C, Cox P, Johnson GR, Maudlin P. A constitutive formulation for anisotropic materials suitable for wave propagation computer program. Comput Mech 1994;15:201-23.

[12] Lempriere BM. Poisson's ratio in orthotropic materials. AIAA J 1968;6(11):2226-7.

[13] Lysmer J, Kuhlemeyer RL. Finite dynamic model for infinite media. J Eng Mech Div, Proc ASCE 1969;95(EM4):859-77.

[14] TrueGrid. Livermore, CA, USA; XYZ Scientific Applications, Inc; 2000.

[15] Justo J, Marques T. Design and testing of composite panels for ballistic protection. In: Second International Symposium on Impact Engineering, Pequim, China, 1996.

[16] Twaron in Soft Body Armour. Wuppertal: AKZO; 1993. 